\title{
Article \\ Foot Contact Dynamics and Fall Risk among Children Diagnosed with Idiopathic Toe Walking
}

\author{
Rahul Soangra ${ }^{1,2} \mathbb{1}$, Michael Shiraishi ${ }^{1}$, Richard Beuttler ${ }^{3}$, Michelle Gwerder ${ }^{3}$, LouAnne Boyd ${ }^{4}$, \\ Venkatesan Muthukumar ${ }^{5}$, Mohamed Trabia ${ }^{5}{ }^{(0)}$, Afshin Aminian ${ }^{6}$ and Marybeth Grant-Beuttler ${ }^{1,6, *}$ \\ 1 Crean College of Health and Behavioral Sciences, Chapman University, Orange, CA 92866, USA; \\ soangra@chapman.edu (R.S.); shiraishi@chapman.edu (M.S.) \\ 2 Fowler School of Engineering, Chapman University, Orange, CA 92866, USA \\ 3 School of Pharmacy, Chapman University, Orange, CA 92618, USA; rbeuttle@chapman.edu (R.B.); \\ michelle.gwerder@hest.ethz.ch (M.G.) \\ 4 Institute of Biomechanics, 8092 ETH Zurich, Switzerland; lboyd@chapman.edu \\ 5 Howard R. Hughes College of Engineering, University of Nevada, Las Vegas, NV 89154, USA; \\ Venkatesan.muthukumar@unlv.edu (V.M.); Mohamed.trabia@unlv.edu (M.T.) \\ 6 Children's Hospital of Orange County, Orange, CA 92868, USA; aaminian@choc.org \\ * Correspondence: beuttler@chapman.edu; Tel.: +1-714-744-7626
}

check for updates

Citation: Soangra, R.; Shiraishi, M.; Beuttler, R.; Gwerder, M.; Boyd, L.; Muthukumar, V.; Trabia, M.; Aminian, A.; Grant-Beuttler, M. Foot Contact Dynamics and Fall Risk among Children Diagnosed with Idiopathic Toe Walking. Appl. Sci. 2021, 11, 2862. https://doi.org/10.3390/app1106 2862

Academic Editor: Nyeonju Kang

Received: 1 February 2021

Accepted: 18 March 2021

Published: 23 March 2021

Publisher's Note: MDPI stays neutral with regard to jurisdictional claims in published maps and institutional affiliations.

Copyright: (c) 2021 by the authors. Licensee MDPI, Basel, Switzerland. This article is an open access article distributed under the terms and conditions of the Creative Commons Attribution (CC BY) license (https:// creativecommons.org/licenses/by/ $4.0 /)$.

\begin{abstract}
Children that are diagnosed with Idiopathic Toe walking (cITW) are characterized by persistent toe-to-toe contacts. The objective of this study was to explore whether typical foot contact dynamics during walking predisposes cITW to a higher risk of falling. Twenty cITW and agematched controls performed typical and toe walking trials. The gait parameters related to foot contact dynamics, vertical force impulses during stance, slip, and trip risk were compared for both groups. We found that cITW manifest less stable gait and produced significantly higher force impulses during push-off. Additionally, we found that cITW had a higher slip-initiation risk that was associated with higher foot contact horizontal and vertical velocities in addition to lower transitional acceleration of center of mass. We found that cITW exhibited a higher trip risk with toe clearance being significantly lower when compared to healthy counterparts. This study allowed for a quantitative description of foot contact dynamics and delineated typical from toe walking among cITW. Overall, the results indicate that cITW are less stable during typical walking and are prone to a higher risk of slip and trip-like falls.
\end{abstract}

Keywords: Idiopathic Toe Walking; fall risk; foot contact dynamics; foot initial contact; push-off

\section{Introduction}

Toe walking is defined as walking on the forefeet as compared to a typical heel-toe gait pattern. Toe touch can be observed in early ambulation, but it is considered to be atypical after three years [1,2]. The prevalence of Idiopathic Toe Walking (ITW) among children has been reported between $2-12 \%$ of the child population [3]. Earlier research has reported an increased fall risk due to frequent tripping and pain in the leg or foot among children diagnosed with Idiopathic Toe Walking (cITW) [1,4]. Some researchers also reported limited ankle dorsiflexion, functional and passive range-of-motion (ROM), which predisposes them to higher fall risk and ankle injuries [5]. The cause of toe walking in ITW may not be clear. However, some researchers emphasize hyperactive reflexes and they have based current therapeutic strategies on this theory in the clinics [6-8].

Clinicians and movement science researchers have sought to understand how toe walking behavior influences fall risk by monitoring the subtle differences in the ground reaction forces (GRF). GRF metrics may reflect internal loading differences among cITW as compared to healthy controls. Toe walking with GRF analysis can potentially reveal factors that influence injury risk, including the foot loading intensity (both high magnitude and 
short duration of loading) as well as development and remodeling in children (influenced by duration of loading and length of rest during toe walking) [9]. Previously, researchers have derived parameters using GRFs, such as peak forces, impulses, temporal events during the stance phase of gait to assess gait pathology [10]. Because toe walking is performed daily and frequently in this population, it is imperative to understand foot loading dynamics, slip and trip risk to intervene in cITW. A quantitative description of foot contact dynamics [11-14] and gait assessment may be helpful in (i) revealing severity of ITW, (ii) establishing norms among cITW, and (iii) devise intervention strategies.

It is well established that, during toe walking, increased plantarflexion can compromise gait stability and it is often associated with decreased walking speed and stride length $[15,16]$. To maintain an upright posture, one has to stabilize the center of mass (COM) over the base of support. Stabilizing the COM over a small base of support when advancing their body forward during walking is challenging for CITW who are described to produce a bouncy gait with higher energy expenditures. Along with inefficient gait, the toe walkers have sensory processing and integration issues $[17,18]$. Sensory integration is defined as the registration and modulation of input sensory signals (somatosensory, vision, and vestibular) to execute the movement. However, several studies have failed to demonstrate an enhanced sensory contribution to the muscle activity in toe walking children, and an alternative theory, which emphasizes altered central control as an adaptation to demands of muscle and joint mechanics, has been suggested [19-21]. It is not well known whether the toe walking observed among cITW is distinct as compared to the toe walking observed in healthy controls. It is also not known if angle of attack during foot initial contact (FIC) differs among cITW and will predispose them to falls. Given the dearth of evidence and inconsistent findings on toe walking among this particular population of ITW, this study's primary objective is to explore kinetic and gait differences during typical and toe walking and investigate how these influences fall risk. This study aims to examine how toe walking influences foot landing dynamics among cITW as compared to their healthy counter-parts and whether it predisposes them to trip risk and slip risk.

\section{Materials and Methods}

Ten cITW (five females, five males; age $=7.5 \pm 2.3$ years, weight $=60.8 \pm 16.4 \mathrm{lbs}$, height $=49.6 \pm 5.8$ inches) and 10 healthy (five females, five males; age $=8.7 \pm 3.4$ years, weight $=69 \pm 33.1 \mathrm{lbs}$, height $=51.2 \pm 9.7$ inches) children participated in this research At recruitment, the diagnosed cITW participants were referred by a pediatric orthopedic surgeon and Orange county area physical therapist. All of the participants signed a written informed consent and the protocol was approved by the Children's Hospital of Orange County (CHOC) Institutional Review Board (IRB\# 170870). CodaMotion 3-D Analysis System (Charnwood Dynamics Ltd., Leicestershire, UK) with four CODA optical sensors were used for data collection. The system captured the vertical, horizontal, and rotational movements by tracking the attached marker positions. The system consisted of infra-red light-emitting diode (LED) markers and drive boxes as marker devices, which were attached to bony anatomical landmarks at the skin. A cluster of four markers were placed at each segment (thigh, shank, upper arm, and pelvis). A total of 22 markers were placed bilaterally on the 5th metatarsal head, the base of the 5th metatarsal, with clusters placed on the shank, thigh, and pelvis. Clusters were used to mark virtual markers at the anterior superior iliac spine (ASIS), femoral head, lateral and medial femoral epicondyle, lateral tibial epicondyle, and medial and lateral malleoli. Codamotion ODIN software suite analyzed the data from sensor modules. The visibility of the markers was monitored in realtime during each walking trial. Functional joint centers, segment angles, and joint moments were computed using ODIN. The GRF data were collected using two forceplates model Bertec BP400600 (Bertec, Columbus, Ohio 43219). The GRF was filtered and normalized prior to evaluating the impact dynamics. For each trial evaluation, individual stance phases were parsed out, and outcome kinetic parameters were computed, as detailed below. The 
whole-body kinematics and GRFs were collected at $100 \mathrm{~Hz}$ and $1000 \mathrm{~Hz}$, respectively, and then low-pass filtered at $6 \mathrm{~Hz}$ cut-off frequency (4th order, zero-lag Butterworth).

Procedures: the participants were asked to perform walking on a $10 \mathrm{~m}$ long walkway at their preferred pace. The walkway and surrounding area were well lit. The two forceplates were embedded at the center of the walkway. The participants were asked to walk in their natural or 'typical' way in which they usually walk at home and outside. The typical gait in cITW may be affected by the new testing environment, and children may consciously present best heel strikes during walking in clinicians' presence. One movement scientist with expertise in pediatrics physical therapy (PT) and two other PT's continuously interacted with the child participant and with parents to acclimatize participants to the new environment, such that the child could present typical walk during data collection. Two PT's stood on each side of the walkway with visual target and performance boards. The boards provided stars for every trial completion. The typical gait is different for cITW and healthy children. cITW usually perform toe-to-toe gait similar to toe walking, whereas healthy children perform a heel-to-toe gait. The second task was to perform toe walking, cITW performed this with higher plantarflexion angles than their typical walk. Each participant walked at least 10 trials of each walking type barefoot. The trial was repeated if the participants did not step at the center of the forceplate, or failed to perform the instructed kind of walk. The investigators visually checked for foot strike on forceplate and marked those trials as good. During data analysis, only three trials were randomly chosen from the trials that were marked as good for each walking type (typical and toe walking). The mean age and gender were balanced in both groups (ITW versus healthy controls).

Parameters from Vertical Ground Reaction Forces (GRF): vertical GRF was obtained when the participants walked over a forceplate that was embedded in the 10-m long walkway center. The vertical GRF was divided into a sequence of events (i) Foot initial Contact (FIC), (ii) Peak Loading Response (PLR), (iii) mid-stance (MS), (iv) peak push off (PPO), and (v) foot off (FO).

Loading rate is defined as the slope of vertical GRF from FIC to PLR. COM ascending rate is defined as the slope of vertical GRF from PLR to MS. COM descending rate is defined as the slope of vertical GRF from MS to PPO. Push off rate is defined as the slope of vertical GRF from PPO to FO. The times taken to reach these events are shown in Figure 1. Similarly, force impulses were computed as area under the GRF-time curve and defined as loading impulse, COM ascending impulse, COM descending impulse, and push-off impulse. The impulses were calculated, as shown by Equation (1) below. Where Vertical $\mathrm{RF}(\mathrm{t})$ is GRF at time $\mathrm{t}$.

$$
\text { Force Impulse }=\int_{\mathrm{t} 1}^{\mathrm{t} 2} \text { Vertical GRF(t).dt }
$$

Joint angles were evaluated at the hip, knee, and ankle. Each segment was represented with three strategically placed markers to compute embedded vector basis (EVB) [22]. EVB was constructed using the Gram-Schmidt Orthogonalization method and embedded axes were aligned to be anatomically meaningful, as per ISB recommendations [22]. In the neutral position, joint angles are zero and they are quantified as segments reposition [23]. The foot segment angle was computed as the angle made by the line adjoining heel and toe markers with the horizontal axis (as shown in Figure 2). 




Figure 1. Stance vertical ground reaction force with events (i) foot initial contact (FIC), (ii) Peak Loading Response (PLR), (iii) mid-stance (MS), (iv) peak push off (PPO), and (v) foot off (FO). Slopes from FIC to PLR was defined as loading rate; PLR to MS as COM ascending rate; MS to PPO as COM descending rate; PPO to FO as Push off rate.

Foot Segment Angles during Foot Initial Contact (FIC)



i) Healthy Typical Walking

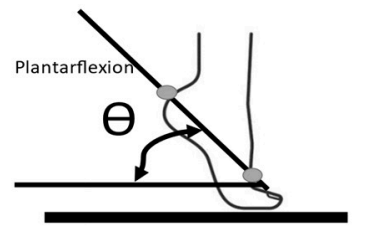

ii) Toe Walking

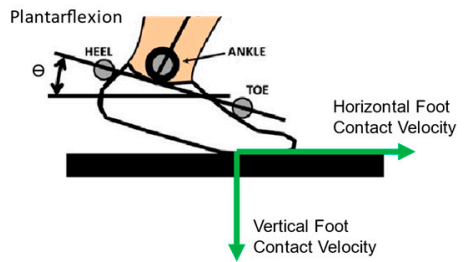

iii) ITW Typical Walking

Figure 2. Foot segment angles during foot initial contact for (i) healthy typical walking, (ii) toe walking, and (iii) Idiopathic Toe Walking (ITW) typical walking. Dorsiflexion angles are taken as positive and plantarflexion as negative.

Gait Assessments: foot contact was defined when the vertical force exceeded more than $7 \mathrm{~N}$ after the foot contacted the ground. In pilot trials, we placed heel and toe markers on lateral sides of the foot, such that foot initial contact determination (forceplate versus motion capture camera system) was consistently at about $7 \mathrm{~N}$ of vertical force. This could be due to signal noise from forceplate, which could not consistently detect force less than $7 \mathrm{~N}$. The foot contact velocity was computed while utilizing the foot position marker at toe or heel, whichever impacts the ground surface during foot landing. The foot contact velocity was evaluated for horizontal and vertical directions through foot lowest marker displacement of 1/100 s before and after the foot contact phase of gait cycle using instantaneous foot velocity formula [24,25], as shown in Equation (2).

$$
\text { Foot velocity }=\frac{(\text { Foot Marker Position }(i+1)-\text { Foot Marker Position }(i-1)}{2 \Delta t}
$$

Minimum Toe Clearance (MTC): it is the minimum vertical distance between the toe marker of the swing foot and the walking surface during swing. MTC is associated with trip-related falls during over ground walking [26-29].

Stride Time: it is the time taken from foot initial contact (FIC) to the next FIC of the same foot [30]. Stride Length: the distance that is covered during a stride time is stride 
length [30]. Transitional acceleration of COM is defined as the acceleration of COM during the foot initial contact [31], and it is represented in Equation (3) (Figure 3a).

$$
\text { Transitional COM Acceleration }=\frac{(\operatorname{COM} \text { Velocity }(\mathrm{i}+1)-\operatorname{COM} \text { Velocity }(\mathrm{i}-1))}{2 \Delta \mathrm{t}}
$$

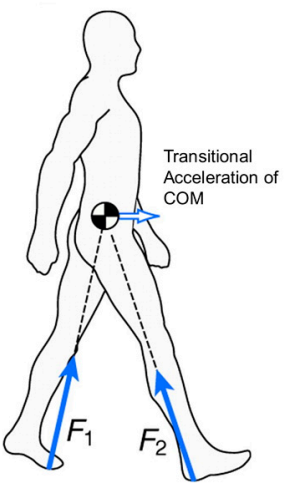

(a)

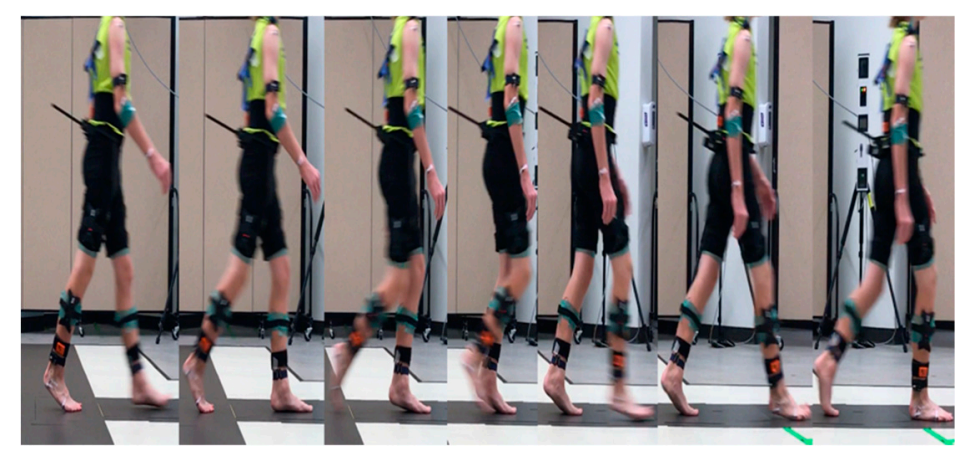

(b)

Figure 3. (a) Transitional acceleration of center of mass (COM) during walking (b) typical walking in cITW participant.

Mixed Factor MANOVA model analysis was conducted while using JMP Software (JMP 15 Pro, SAS Institute Inc., Cary, NC, USA), where the groups and walking type were fixed with subjects as random effects. The significance was set at $p=0.05$. Post Hoc analysis was done using Tukey's HSD. We investigated multiple continuous dependent variables, and MANOVA bundles them together into a weighted linear combination. MANOVA will also compare if the combination differs by the different groups (typical, ITW) and levels of the independent variables (toe walking, typical walking). The model was tested for multicollinearity, normality, and homogeneity of variance. MANOVA was selected, since it has a greater statistical power than regular ANOVA. It can also limit the joint error rate as joint probability of rejecting a true null hypothesis increases with each additional test.

\section{Results}

\subsection{Kinetic Data Analysis}

There was no significant difference between groups (ITW versus healthy controls) across variables (i) loading rate, (ii) COM ascending rate, and (iii) COM descending rate. However, significant interaction effects were found between groups for push-off rate $(p<0.01)$ (Figure 3b). ITW were found to have significantly higher push-off rates during typical walking and significantly lower push off rate during toe walking when compared to healthy counterparts (Figure 4).

Similar results were found for force impulses during vertical GRF. No significant differences were observed for loading impulse, COM ascending impulse, and COM descending impulse, but interaction effects were found between groups and walking type for push-off impulse $(p<0.01)$. However, rates and force impulses for loading, COM ascending, COM descending, and push off were significantly different for the two walking types (typical versus toe walking) when combining all participants from both groups. The ankle, knee, and hip angles during stance events in cITW and controls have been reported for toe walking and typical gait (Table 1). 


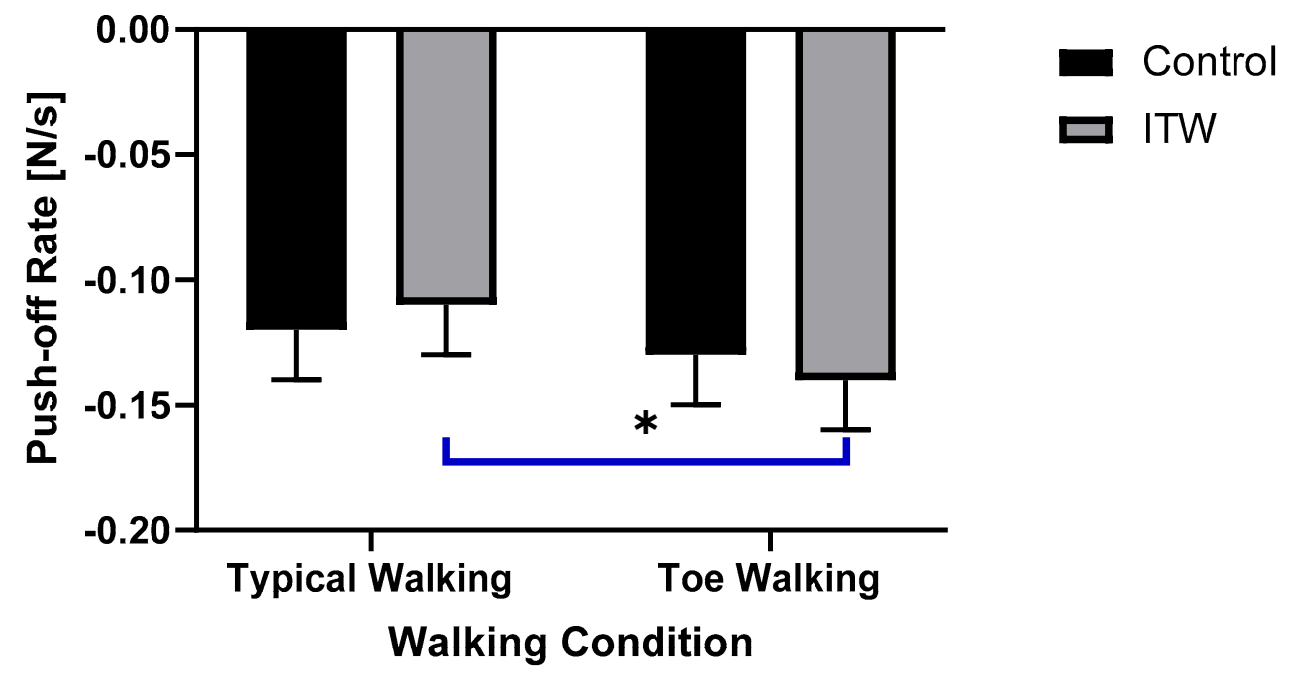

Figure 4. Push-off rate among ITW and Controls during typical and toe walking.

Table 1. Foot segment angle at FIC and FO and joint angles at loading, midstance, and push-off among ITW and controls. Here significant differences are represented by $*(p<0.05)$.

\begin{tabular}{ccccc}
\hline & \multicolumn{2}{c}{ Control } & ITW & Typical \\
\hline Angles [Degrees] & Toe Walking & $\begin{array}{c}\text { Typical } \\
\text { Walking }\end{array}$ & Toe Walking & Walking \\
\hline Foot angle at FIC & $-26.0 \pm 17.1$ & $1.6 \pm 23.8$ & $-29.1 \pm 17.5$ & $-16.3 \pm 24.1$ \\
Foot angle at Foot Off (FO) & $-43.2 \pm 36.0$ & $-41.1 \pm 37.8$ & $-47.2 \pm 35.0$ & $-34.0 \pm 35.2$ \\
Ankle angle at Loading & $-19.2 \pm 16.9$ & $-9.1 \pm 13.4$ & $-24.5 \pm 16.6$ & $-15.6 \pm 12.7$ \\
Ankle angle at Midstance & $-16.4 \pm 8.7$ & $3.7 \pm 6.2$ & $-22.8 \pm 8.0$ & $-6.2 \pm 10.9$ \\
Ankle angle at Push-off & $-25.8 \pm 12.0$ & $4.6 \pm 8.8$ & $-27.7 \pm 9.1$ & $-4.0 \pm 11.5$ \\
Knee angle at Loading & $25.2 \pm 12.8$ & $32.0 \pm 14.2$ & $25.5 \pm 9.7$ & $31.9 \pm 16.4$ \\
Knee angle at Midstance & $28.8 \pm 23.0$ & $30.2 \pm 23.9$ & $30.1 \pm 23.3$ & $38.5 \pm 28.0$ \\
Knee angle at Push-off * & $6.6 \pm 13.0$ & $4.1 \pm 7.8$ & $6.8 \pm 8.9$ & $11.1 \pm 9.8$ \\
Hip angle at Loading & $23.0 \pm 36.6$ & $24.7 \pm 20.3$ & $26.4 \pm 22.9$ & $25.2 \pm 26.2$ \\
\hline Hip angle at Midstance & $19.3 \pm 41.5$ & $24.9 \pm 16.0$ & $28.9 \pm 34.9$ & $33.1 \pm 20.3$ \\
Hip angle at Push-off & $14.1 \pm 39.4$ & $14.1 \pm 22.7$ & $19.2 \pm 39.9$ & $28.2 \pm 23.8$ \\
\hline
\end{tabular}

The time to reach mid-stance from foot initial contact was found to be significantly longer for toe walking when compared to typical walking for both groups $(p=0.0001)$. We found the time taken from push-off to foot off was significantly longer among ITW during their typical walking than toe walking $(p=0.002)$. We found that the ITW group produced significantly higher joint angles at the knee during push-off phase of typical walking $(p=0.03)$ as compared to healthy counterparts (Figure 5). Foot segment angles were significantly different for controls during foot initial contact $(p=0.04)$ (Figure 6). It was found that the foot dorsiflexed during FIC when typical walking and plantarflexed during FIC during toe walking. The joint angles at ankle during midstance were found to be significantly lower among ITW group as compared to the controls $(p=0.006)$.

We also found that during toe walking, joint angles at loading, mid-stance, and push-off were found to be significantly lower than typical walking among both groups $(p=0.0005)$.

\subsection{Gait Assessments}

The interaction effects were found to be significant $(p<0.001)$ for stride length between walking type (toe walking versus typical), and groups (ITW versus controls). Tukey's HSD revealed that controls produced longer strides during typical walking. Contrastingly cITW made longer strides during toe walking. Similarly, we found that the stride times were shorter during toe walking among both groups. 




Figure 5. Knee angles during push-off among controls versus cITW. Where ${ }^{*}$ represents significant difference with $p<0.05$.

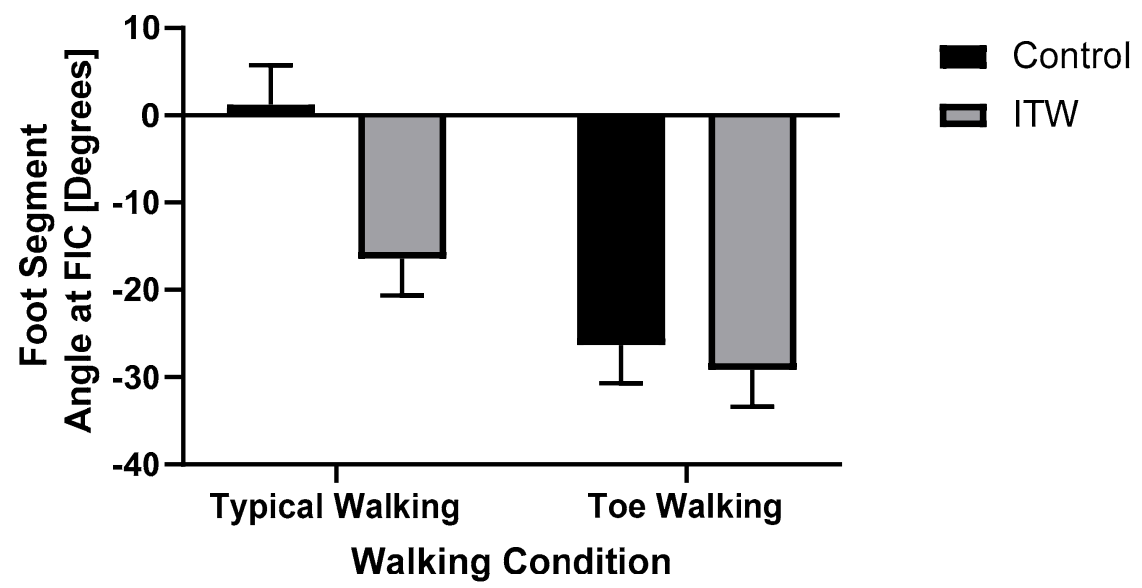

Figure 6. Foot segment angle at foot initial contact, dorsiflexion (positive), and plantarflexion (negative).

We found significant interaction effects $(p=0.01)$ for single stance time (SST). We found that cITW had longer SST during toe walking when compared to typical walking (Figure 7). Toe walking resulted in increased step width as compared to typical gait for both groups $(p=0.0004)$.

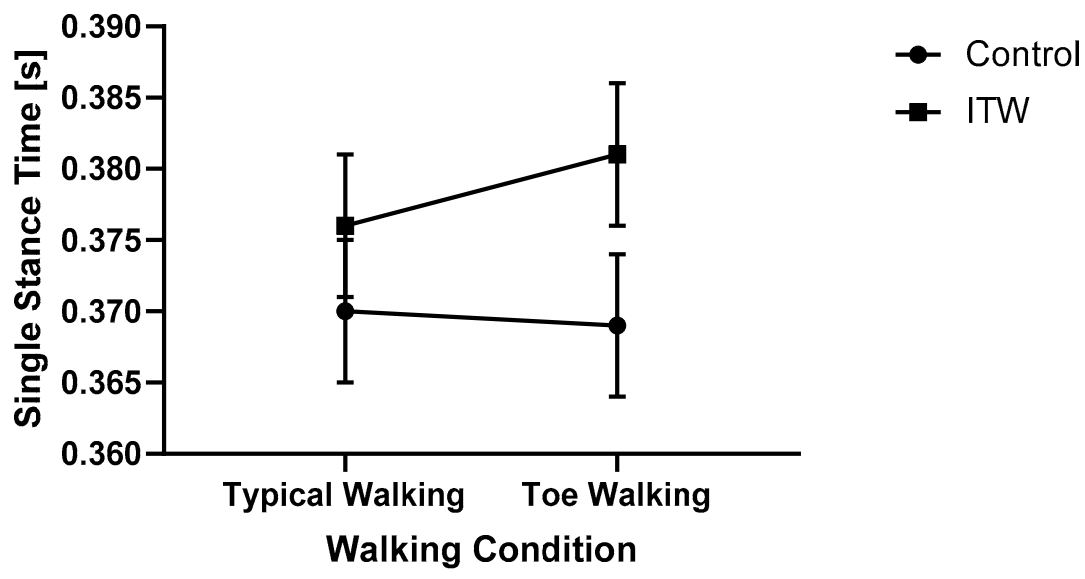

Figure 7. Interaction effects were seen in single stance time for group versus walking type. 
Toe clearance significantly decreased among ITW during typical gait when compared to toe walking $(p=0.005)$ (Figure 8$)$.

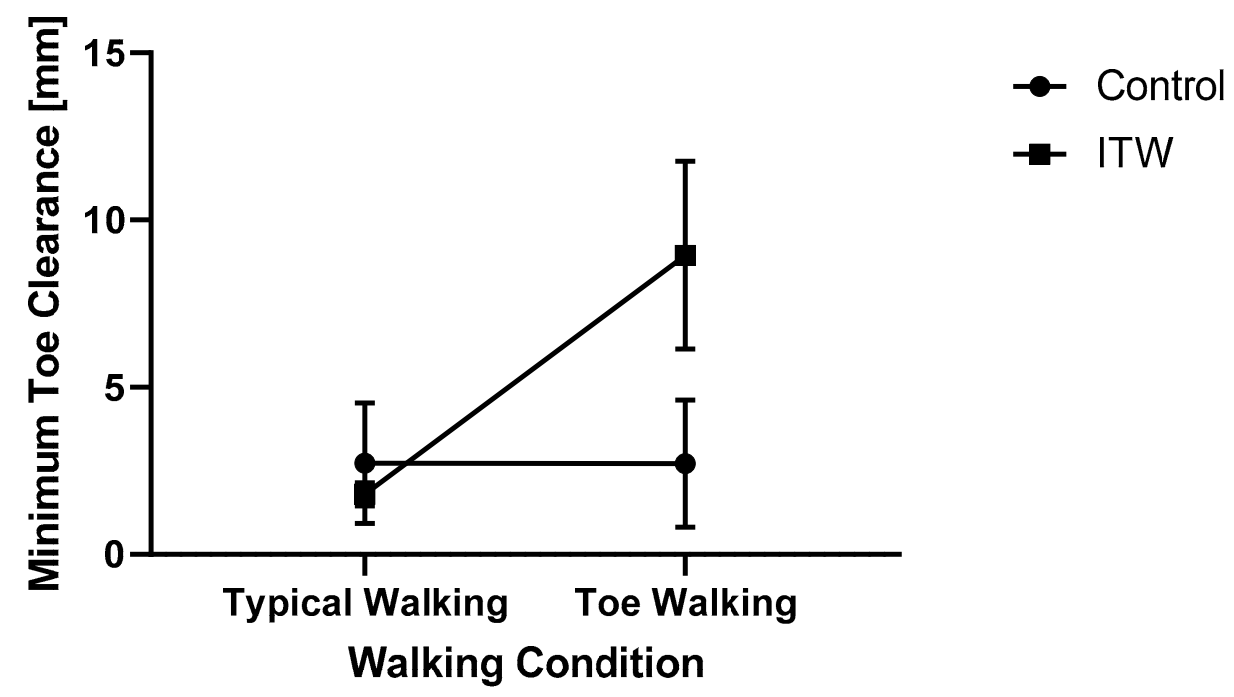

Figure 8. Interaction effects were seen in minimum toe clearance among groups and walking type.

Vertical foot contact velocity was significantly higher during toe walking among both of the groups $(p=0.0004)$ as compared to typical gait. Vertical foot contact velocity was the lowest among controls during typical gait. Horizontal foot contact velocity was found to be significantly higher during toe walking than typical walking among controls $(p=0.0006)$ (Figure 9).
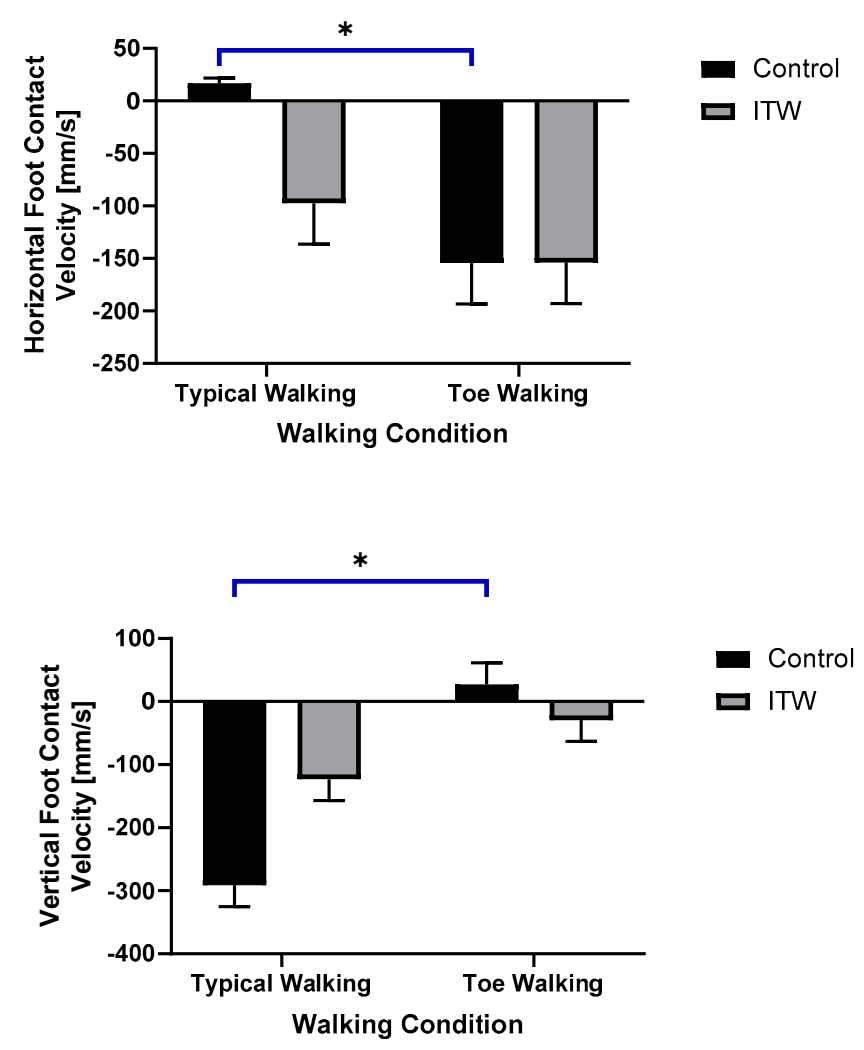

Figure 9. Horizontal and vertical foot contact velocity during typical and toe walking. Where* represents significant difference with $p<0.05$. 
Transitional Acceleration of COM was found lower among ITW during typical walking, but it was lower among the controls during toe walking. Table 2 reports all of these gait parameters for both the cITW and control group. In addition, mean joint angles during stance are reported for push off, swing, and FIC events shown in Table 3.

Table 2. Gait parameters associated with ITW and control. Here significant differences are represented by * $(p<0.05)$.

\begin{tabular}{|c|c|c|c|c|}
\hline & \multicolumn{2}{|c|}{ Control } & \multicolumn{2}{|c|}{ ITW } \\
\hline & Toe Walking & Typical Walking & Toe Walking & Typical Walking \\
\hline Stride Length * $[\mathrm{mm}]$ & $921.4 \pm 197.5$ & $1066.0 \pm 237.1$ & $980.5 \pm 169.3$ & $911.2 \pm 145.1$ \\
\hline Stride Time $[\mathrm{s}]$ & $0.8 \pm 0.1$ & $0.9 \pm 0.1$ & $0.8 \pm 0.07$ & $0.9 \pm 0.1$ \\
\hline Single Stance Duration [s] & $0.3 \pm 0.03$ & $0.3 \pm 0.01$ & $0.3 \pm 0.01$ & $0.3 \pm 0.03$ \\
\hline Minimum Toe Clearance [mm] & $2.7 \pm 10.5$ & $2.7 \pm 11.1$ & $8.9 \pm 17.1$ & $0.3 \pm 13.1$ \\
\hline Step Width [mm] & $147.0 \pm 38.9$ & $133.6 \pm 28.6$ & $167.3 \pm 41.3$ & $145.2 \pm 47.4$ \\
\hline Horizontal Foot Contact Velocity [mm/s] & $-154.3 \pm 316.4$ & $16.7 \pm 176.7$ & $-47.7 \pm 220.0$ & $-97.4 \pm 231.4$ \\
\hline Vertical Foot Contact Velocity * $[\mathrm{mm} / \mathrm{s}]$ & $27.3 \pm 172.1$ & $-291.2 \pm 360.9$ & $-29.3 \pm 108.1$ & $-123.1 \pm 211.0$ \\
\hline Transitional Acceleration of COM * $\left[\mathrm{mm} / \mathrm{s}^{2}\right]$ & $-288.0 \pm 4771.5$ & $782.94 \pm 2631.8$ & $1284.0 \pm 3636.5$ & $99.964 \pm 2366.8$ \\
\hline
\end{tabular}

Table 3. Mean \pm SD of Joint angles during push-off, swing and FIC for ankle, knee and hip for ITW and control with different walking types.

\begin{tabular}{ccccccccc}
\hline & & & Toe Walking & & \multicolumn{3}{c}{ Typical Walking } \\
\hline & & Push-Off & Swing & FIC & Push-Off & Swing & FIC \\
\hline \multirow{2}{*}{$\begin{array}{c}\text { Control } \\
{[\text { Degrees] }}\end{array}$} & Ankle & $-26.8 \pm 12.4$ & $-34.2 \pm 8.0$ & $-27.6 \pm 10.3$ & $1.8 \pm 5.9$ & $-15.4 \pm 6.4$ & $0.2 \pm 5.5$ \\
\cline { 2 - 8 } & Knee & $5.1 \pm 9.8$ & $25.6 \pm 6.4$ & $4.4 \pm 8.7$ & $1.5 \pm 7.2$ & $33.5 \pm 6.6$ & $1.7 \pm 7.4$ \\
\cline { 2 - 8 } & Hip & $38.0 \pm 14.5$ & $3.0 \pm 12.9$ & $36.3 \pm 13.6$ & $37.6 \pm 12.8$ & $2.2 \pm 10.7$ & $37.6 \pm 11.3$ \\
\hline \multirow{2}{*}{$\begin{array}{c}\text { ITW } \\
\text { [Degrees] }\end{array}$} & Knee & $1.4 \pm 8.4$ & $22.1 \pm 5.7$ & $1.8 \pm 7.5$ & $0.8 \pm 5.0$ & $32.2 \pm 6.0$ & $1.8 \pm 5.1$ \\
\cline { 2 - 8 } & Hip & $41.3 \pm 15.2$ & $4.5 \pm 11.0$ & $42.7 \pm 13.8$ & $38.7 \pm 11.8$ & $8.7 \pm 12.2$ & $40.0 \pm 11.7$ \\
\hline
\end{tabular}

\section{Discussion}

Our major findings reveal that cITW are more prone to falls due to (i) lower toe clearance (increased trip risk), (ii) high horizontal heel velocity along with reduced transitional acceleration of COM (slip initiation risk), and (iii) reduced stability when stiffening during stance. We also found cITW typical walking to be inherently inefficient due to several reasons, such as (i) significantly higher push-off impulses, (ii) more knee flexion angles during stance, (iii) high vertical heel velocity, and (iv) already plantarflexed ankle fail to produce force generation capacity during push-off. These results have several broader impacts, for example, (i) foot segment angles and peak-push of impulses could possibly reveal critical insights into severity of ITW, (ii) since no previous study has established norms for foot contact dynamics among cITW population this study will serve as background work, (iii) new interventions, such as providing haptic feedback during toe walking [32] and identifying toe walking utilizing artificial intelligence [33], will help to develop novel interventions.

Toe walking may be associated with several other disorders and diagnoses, such as cerebral palsy (CP), muscular dystrophy, autism, myopathy, mental retardation, childhood schizophrenia, and muscular dystrophy [1,34,35]. Some researchers claim that ITW children do not have neurological signs [34], but only clinical signs of limited active dorsiflexion during gait. Others claim that ITW may be due to unknown deficits in the central nervous system or a neuropathic process as in muscle properties [36]. ITW children have been reported to have delayed the corticospinal tract [35]. Idiopathic toe walking is commonly diagnosed in pediatric orthopedic clinics as benign and it is often not informed to parents as a significant concern. If the complication gets aggravated with injuries, treatments, such as botox, casting, or ankle-foot orthotics (AFO), or even orthopedic surgery, is often 
recommended. Scientific knowledge lacks an objective assessment of ITW foot contact dynamics and how these influence slip and trip risk during walking as compared to agematched healthy counterparts. In this study, we investigated ITW gait using kinetics and gait kinematics when walking in two different styles: (i) their usual or typical walking style and (ii) toe walking. Some advantages of using GRF in toe walking analysis include ease of use, non-invasive, portable forceplates, and they can be easily installed in clinical walking environments. The assessment of gait characteristics and risk of falls among cITW is challenging, since children can alter their gait while under observation in clinical laboratory. This makes gait analysis among ITW difficult with gait and fall risk underdiagnosed and underappreciated. To our knowledge, this is the first study investigating typical and toe walking differences among ITW and the age matched control population. The major findings are adaptations of cITW during push-off by producing significantly higher pushoff rates and force impulses. They are also found to exhibit significantly higher joint angles at the knee level during the push-off. This adaptation at the knee joint through increased flexion angles during typical walking could be an extra burden on cITW and induce muscle fatigue and increase the risk of injuries.

Earlier researchers have used a kinematic analysis of ankle range of motion to differentiate ITW from CP [16,37-39]. We found that both ITW and control groups demonstrated significantly lower joint angles at loading, mid-stance, and push-off during toe walking (Table 1), suggesting rigidity at the lower extremity. The stiffening behavior and freezing the degree of freedom at the lower extremity joints could jeopardize gait stability and increase the chances of severe falls and injuries $[40,41]$. It was also found that foot segment angles at FIC were in the position of dorsiflexion for control typical walking, whereas it was at plantarflexion angle for cITW similar to toe walking (Figure 6). The magnitude of plantarflexion angles during FIC could serve as a score of ITW severity.

We also found that stride length was significantly longer during typical walking among both groups and shorter during toe walking. The reduced stride length indicates that toe walking is more restrictive with a limited degree of freedom to both groups and it will redistribute plantar foot pressures [42] to maintain COM over a smaller base of support. The single-limb stance time of gait is important, since, during this time, the foot is on the ground supporting the gravitational load of the whole body and propelling the $\mathrm{COM}$ forward, i.e., acting against the inertial and frictional load. A wide variety of force receptors are activated during stance, including cutaneous receptor, high threshold force receptor, and spindle from ankle joint muscles [43-45], to provide force feedback through afferent pathways [46]. The increased stance durations among cITW could adapt to acquire more proprioceptive and force feedback information to maintain stability compromising minimum plantar contact area with the ground. This compensation for stability is partially accommodated with increased step width, as found in our study. Although cITW seem to be exhibit coordinated walking, but their plantar weight-bearing is at the forefront of the foot, with plantarflexion ranging from 2.7 to 28.3 degrees during typical walking (Table 1). Ankle plantarflexion strength is primarily a function of moment arm from ankle joint to the ground reaction force line, acting at the center of pressure of the foot. During typical walking among the ITW group, the requirement for ankle plantarflexion is greatest in push-off. However, due to the already plantarflexed ankle, the force-generating capacity of ankle muscles reduces due to the limited range available for ankle plantarflexion. The neuromuscular demand on plantar flexors is greater for cITW [47-50]. These neuromuscular demands increase with an increase in moment arm during typical waling in cITW [51].

We also found that cITW had significantly lower toe clearance values during typical walking. A lower toe clearance has been associated with trip-related stumbles [52], and it imposes the highest risk of unintentional contact with obstacles or the ground [53]. This finding is important, since no previous study has reported trip risk among cITW. Along with trip risk, we evaluated slip initiation risk through foot contact velocity and transitional acceleration of COM. The horizontal foot contact velocity is reduced in healthy adults through the activation of the hamstring [25], but this stabilizing reduction in velocity was 
not observed in the ITW population (Table 2 and Figure 9). Thus, high horizontal heel contact velocity increases the slip initiation risk similarly to older adults. In addition, cITW are found to take a significantly longer time to reach mid-stance after foot initial contact during typical walking. This may have attributed to the significantly lower transitional acceleration of COM in ITW population. Transitional acceleration of COM has been reported to influence slip initiation (by modulating friction demand) [54,55]. The fall risk increases with the reduced acceleration of COM during walking. A repetitive high vertical foot contact velocity could increase the risk of injury due to the high ground impact.

We found that foot segment angles were significantly lower for ITW than controls (1.6 $6^{\circ}$ dorsiflexion as compared to $16.3^{\circ}$ plantarflexion). This was attributed to higher horizontal foot contact velocity among cITW. Researchers have previously established a relationship with horizontal foot contact velocity and hamstring activation rate, ultimately leading to slip-induced fall accidents [25]. It is known that older adults have higher foot contact velocity when compared to younger adults due to slower hamstring activation rate, thereby modulating friction demand at the foot-floor interface and ultimately leading to increased likelihood of slip induced falls [25,31]. One potential limitation of this study is to acclimatize child participants to produce typical gait. However, the experiments included trained PT's, who continuously encouraged participants to maintain their typical gait by encouraging them to look forward at the target while walking and incentivizing through drawing stars once completing a walking trial.

\section{Conclusions}

Overall, this research has broader impacts in understanding fall risk and developing future novel personalized interventions for cITW using wearable sensors [32] and artificial intelligence [33]. This study will help to interpret gait characterization of ITW gait patterns, which would allow us to delineate using deviations that originate from ITW's typical walking versus induced toe-walking, both requiring different nervous control for compensatory adaptations. These adaptations are difficult to visualize by the naked eye, since subtle mechanical output during a movement in the multijointed human biomechanical system affects the whole lower extremity due to mechanical coupling. As per our knowledge, this is the first study looking into slip and trip risk variables that have considerable implications for clinicians and movement scientists in classifying the severity of toe walking and designing novel intervention tools for ITW.

Author Contributions: Conceptualization, R.S., R.B. and M.G.-B.; methodology, M.S., M.G.; software, M.S. and R.B.; validation, R.S., M.S., R.B., M.G.-B. and M.G.; formal analysis, R.B.; investigation, R.S., R.B., and M.G.-B.; resources, M.G.-B.; data curation, M.S.; writing-original draft preparation, R.S.; writing-review and editing, L.B., V.M., M.T., A.A.; visualization, R.S.; supervision, M.G.-B.; project administration, R.S., M.G.-B.; funding acquisition, M.G.-B. All authors have read and agreed to the published version of the manuscript.

Funding: This research was funded by Kay Family Foundation and CHOC-Transformational Philanthropic Venture Funding Program and The APC was funded by CHOC.

Institutional Review Board Statement: The study was conducted according to the guidelines of the Declaration of Helsinki, and approved by the Institutional Review Board (or Ethics Committee) of CHOC (IRB\#170870 protocol code and 08/2017 as date of approval).

Informed Consent Statement: Informed consent was obtained from all subjects involved in the study. Written informed consent has been obtained from the patient(s) to publish this paper.

Acknowledgments: We are thankful to Christopher Hoang, and DPT students during data collection.

Conflicts of Interest: The authors declare no conflict of interest. 


\section{References}

1. Sobel, E.; Caselli, M.; Velez, Z. Effect of persistent toe walking on ankle equinus. Analysis of 60 idiopathic toe walkers. J. Am. Podiatr. Med. Assoc. 1997, 87, 17-22. [CrossRef]

2. Sutherland, D.H.; Olshen, R.; Cooper, L.; Woo, S.L. The development of mature gait. J. Bone Jt. Surg. Am. 1980, 62, 336-353. [CrossRef]

3. Fox, A.; Deakin, S.; Pettigrew, G.; Paton, R. Serial casting in the treatment of idiopathic toe-walkers and review of the literature. Acta Orthop. Belg. 2006, 72, 722-730.

4. Hirsch, G.; Wagner, B. The natural history of idiopathic toe-walking: A long-term follow-up of fourteen conservatively treated children. Acta Paediatr. 2004, 93, 196-199. [CrossRef]

5. Tabrizi, P.; McIntyre, W.M.J.; Quesnel, M.B.; Howard, A.W. Limited dorsiflexion predisposes to injuries of the ankle in children. J. Bone Jt. Surg. Br. 2000, 82, 1103-1106. [CrossRef]

6. Tardieu, C.; Lespargot, A.; Tabary, C.; Bret, M.D. Toe-walking in children with cerebral palsy: Contributions of contracture and excessive contraction of triceps surae muscle. Phys. Ther. 1989, 69, 656-662. [CrossRef] [PubMed]

7. Gross, R.; Leboeuf, F.; Hardouin, J.B.; Perrouin-Verbe, B.; Brochard, S.; Rémy-Néris, O. Does muscle coactivation influence joint excursions during gait in children with and without hemiplegic cerebral palsy? Relationship between muscle coactivation and joint kinematics. Clin. Biomech. 2015, 30, 1088-1093. [CrossRef]

8. Kedem, P.; Scher, D.M. Foot deformities in children with cerebral palsy. Curr. Opin. Pediatr. 2015, 27, 67-74. [CrossRef]

9. Edwards, W.B. Modeling overuse injuries in sport as a mechanical fatigue phenomenon. Exerc. Sport Sci. Rev. 2018, 46, 224-231. [CrossRef]

10. Chao, E.; Laughman, R.; Schneider, E.; Stauffer, R. Normative data of knee joint motion and ground reaction forces in adult level walking. J. Biomech. 1983, 16, 219-233. [CrossRef]

11. Rodda, J.M.; Graham, H.K.; Carson, L.; Galea, M.P.; Wolfe, R. Sagittal gait patterns in spastic diplegia. J. Bone Jt. Surg. Br. 2004, 86, 251-258. [CrossRef]

12. Winters, T.F., Jr.; Gage, J.R.; Hicks, R. Gait patterns in spastic hemiplegia in children and young adults. J. Bone Jt. Surg. Am. 1987, $69,437-441$.

13. Lin, C.-J.; Guo, L.-Y.; Su, F.-C.; Chou, Y.-L.; Cherng, R.-J. Common abnormal kinetic patterns of the knee in gait in spastic diplegia of cerebral palsy. Gait Posture 2000, 11, 224-232. [CrossRef]

14. Armand, S.; Watelain, E.; Mercier, M.; Lensel, G.; Lepoutre, F.-X. Identification and classification of toe-walkers based on ankle kinematics, using a data-mining method. Gait Posture 2006, 23, 240-248. [CrossRef]

15. Davids, J.R.; Foti, T.; Dabelstein, J.; Bagley, A. Voluntary (normal) versus obligatory (cerebral palsy) toe-walking in children: A kinematic, kinetic, and electromyographic analysis. J. Pediatr. Orthop. 1999, 19, 461-469. [CrossRef] [PubMed]

16. Hicks, R.; Durinick, N.; Gage, J.R. Differentiation of idiopathic toe-walking and cerebral palsy. J. Pediatr. Orthop. 1988, 8, 160-163. [CrossRef]

17. Chu, V.; Anderson, L. Sensory-processing differences in children with idiopathic toe walking (ITW). Am. J. Occup. Ther. 2020, 74, 7411505130. [CrossRef]

18. Williams, C.M.; Tinley, P.; Curtin, M. Idiopathic toe walking and sensory processing dysfunction. J. Foot Ankle Res. 2010, 3, 16. [CrossRef]

19. Berger, W.; Quintern, J.; Dietz, V. Pathophysiology of gait in children with cerebral palsy. Electroencephalogr. Clin. Neurophysiol. 1982, 53, 538-548. [CrossRef]

20. Gough, M.; Shortland, A.P. Could muscle deformity in children with spastic cerebral palsy be related to an impairment of muscle growth and altered adaptation? Dev. Med. Child Neurol. 2012, 54, 495-499. [CrossRef]

21. Willerslev-Olsen, M.; Andersen, J.B.; Sinkjaer, T.; Nielsen, J.B. Sensory feedback to ankle plantar flexors is not exaggerated during gait in spastic hemiplegic children with cerebral palsy. J. Neurophysiol. 2014, 111, 746-754. [CrossRef]

22. Wu, G.; Siegler, S.; Allard, P.; Kirtley, C.; Leardini, A.; Rosenbaum, D.; Whittle, M.; D’Lima, D.D.; Cristofolini, L.; Witte, H.; et al. ISB recommendation on definitions of joint coordinate system of various joints for the reporting of human joint motion-part I: Ankle, hip, and spine. J. Biomech. 2002, 35, 543-548. [CrossRef]

23. Grood, E.S.; Suntay, W.J. A joint coordinate system for the clinical description of three-dimensional motions: Application to the knee. J. Biomech. Eng. 1983, 105, 136-144. [CrossRef] [PubMed]

24. Winter, D.A. Biomechanics and Motor Control of Human Movement, 4th ed.; Wiley: Hoboken, NJ, USA, 2009.

25. Lockhart, T.E.; Kim, S. Relationship between hamstring activation rate and heel contact velocity: Factors influencing age-related slip-induced falls. Gait Posture 2006, 24, 23-34. [CrossRef]

26. Barrett, R.; Mills, P.; Begg, R. A systematic review of the effect of ageing and falls history on minimum foot clearance characteristics during level walking. Gait Posture 2010, 32, 429-435. [CrossRef]

27. Khandoker, A.H.; Lynch, K.; Karmakar, C.K.; Begg, R.K.; Palaniswami, M. Regulation of minimum toe clearance variability in the young and elderly during walking on sloped surfaces. In Proceedings of the 29th Annual International Conference of the IEEE Engineering in Medicine and Biology Society, Lyon, France, 23-26 August 2007; pp. 4887-4890.

28. Murray, M.P.; Clarkson, B.H. The vertical pathways of the foot during level walking. I. Range of variability in normal men. Phys. Ther. 1966, 46, 585-589. [CrossRef] 
29. Nagano, H.; Begg, R.; Sparrow, W.A. Computation method for available response time due to tripping at minimum foot clearance. In Proceedings of the 35th Annual International Conference of the IEEE Engineering in Medicine and Biology Society (EMBC), Osaka, Japan, 3-7 July 2013; pp. 4899-4902. [CrossRef]

30. Hollman, J.H.; McDade, E.M.; Petersen, R.C. Normative spatiotemporal gait parameters in older adults. Gait Posture 2011, 34, 111-118. [CrossRef] [PubMed]

31. Lockhart, T.E.; Woldstad, J.C.; Smith, J.L. Effects of age-related gait changes on the biomechanics of slips and falls. Ergonomics 2003, 46, 1136-1160. [CrossRef] [PubMed]

32. Pollind, M.; Soangra, R.; Grant-Beuttler, M.; Aminian, A. Customized wearable sensor-based insoles for gait re-training in idiopathic toe walkers. Biomed. Sci. Instrum. 2019, 55, 192-198.

33. Kim, S.; Soangra, R.; Grant-Beuttler, M.; Aminian, A. Wearable sensor-based gait classification in idiopathic toe walking adolescents. Biomed. Sci. Instrum. 2019, 55, 178-185.

34. Sala, D.A.; Shulman, L.H.; Kennedy, R.F.; Grant, A.D.; Chu, M.L. Idiopathic toe-walking: A review. Dev. Med. Child. Neurol. 1999, 41, 846-848. [CrossRef] [PubMed]

35. A Caselli, M.; Rzonca, E.C.; Lue, B.Y. Habitual toe-walking: Evaluation and approach to treatment. Clin. Podiatr. Med. Surg. 1988, 5, 547-559.

36. Eastwood, D.M.; Dennett, X.; Shield, L.K.; Dickens, D.R.V. Muscle abnormalities in idiopathic toe-walkers. J. Pediatr. Orthop. B 1997, 6, 215-218. [CrossRef]

37. Rose, J.; Martin, J.G.; Torburn, L.; Rinsky, L.A.; Gamble, J.G. Electromyographic differentiation of diplegic cerebral palsy from idiopathic toe walking: Involuntary coactivation of the quadriceps and gastrocnemius. J. Pediatr. Orthop. 1999, 19, 677-682. [CrossRef]

38. Policy, J.F.; Torburn, L.; Rinsky, L.A.; Rose, J. Electromyographic test to differentiate mild diplegic cerebral palsy and idiopathic toe-walking. J. Pediatr. Orthop. 2001, 21, 784-789. [CrossRef] [PubMed]

39. Kelly, I.P.; Jenkinson, A.; Stephens, M.; O’Brien, T. The kinematic patterns of toe-walkers. J. Pediatr. Orthop. 1997, 17, 478-480. [CrossRef]

40. Choi, H.S.; Baek, Y.S. Effects of the degree of freedom and assistance characteristics of powered ankle-foot orthoses on gait stability. PLoS ONE 2020, 15, e0242000. [CrossRef]

41. Chen, S.K.; Voaklander, D.; Perry, D.; Jones, C.A. Falls and fear of falling in older adults with total joint arthroplasty: A scoping review. BMC Musculoskelet Disord. 2019, 20, 1-8. [CrossRef]

42. Allet, L.; Ijzerman, H.; Meijer, K.; Willems, P.; Savelberg, H. The influence of stride-length on plantar foot-pressures and joint moments. Gait Posture 2011, 34, 300-306. [CrossRef]

43. Cleland, C.L.; Hayward, L.; Rymer, W.Z. Neural mechanisms underlying the clasp-knife reflex in the cat. II. Stretch-sensitive muscular-free nerve endings. J. Neurophysiol. 1990, 64, 1319-1330. [CrossRef]

44. Cleland, C.L.; Rymer, W.Z.; Edwards, F.R.; Gabrielli, B.; Roy, L.; Maller, J. Force-sensitive interneurons in the spinal cord of the cat. Science 1982, 217, 652-655. [CrossRef] [PubMed]

45. Rymer, W.Z.; Houk, J.C.; Crago, P.E. Mechanisms of the clasp-knife reflex studied in an animal model. Exp. Brain Res. 1979, 37, 93-113. [CrossRef] [PubMed]

46. Duysens, J.; Clarac, F.; Cruse, H. Load-regulating mechanisms in gait and posture: Comparative aspects. Physiol. Rev. 2000, 80, 83-133. [CrossRef] [PubMed]

47. Neptune, R.R.; Burnfield, J.M.; Mulroy, S.J. The neuromuscular demands of toe walking: A forward dynamics simulation analysis. J. Biomech. 2007, 40, 1293-1300. [CrossRef] [PubMed]

48. Gravel, D.; Richards, C.L.; Filion, M. Influence of contractile tension development on dynamic strength measurements of the plantarflexors in man. J. Biomech. 1988, 21, 89-96. [CrossRef]

49. Nistor, L.; Markhede, G.; Grimby, G. A technique for measurements of plantar flexion torque with the Cybex II dynamometer. Scand. J. Rehabil. Med. 1982, 14, 163-166.

50. Sale, D.; Quinlan, J.; Marsh, E.; McComas, A.J.; Bélanger, A.Y. Influence of joint position on ankle plantarflexion in humans. J. Appl. Physiol. 1982, 52, 1636-1642. [CrossRef]

51. Rugg, S.; Gregor, R.; Mandelbaum, B.; Chiu, L. In vivo moment arm calculations at the ankle using magnetic resonance imaging (MRI). J. Biomech. 1990, 23, 495-501. [CrossRef]

52. Rosenblatt, N.J.; Bauer, A.; Grabiner, M.D. Relating minimum toe clearance to prospective, self-reported, trip-related stumbles in the community. Prosthetics Orthot. Int. 2017, 41, 387-392. [CrossRef]

53. Killeen, T.; Easthope, C.S.; Demkó, L.; Filli, L.; Lőrincz, L.; Linnebank, M.; Curt, A.; Zörner, B.; Bolliger, M. Minimum toe clearance: Probing the neural control of locomotion. Sci. Rep. 2017, 7, 1922. [CrossRef]

54. Lockhart, T.E.; Woldstad, J.C.; Smith, J.L. Relationship between transitional acceleration of the whole body center-of-mass and friction demand characteristic during gait. In Proceedings of the Human Factors and Ergonomics Society Annual Meeting, Baltimore, MD, USA, 29 September-4 October 2002; SAGE Publications: Thousand Oaks, CA, USA, 2002; Volume 46, pp. 1186-1190.

55. Kim, S.; Lockhart, T.; Yoon, H.-Y. Relationship between age-related gait adaptations and required coefficient of friction. Saf. Sci. 2005, 43, 425-436. [CrossRef] [PubMed] 